\title{
On the Fundamental Particles and Reactions of Nature
}

\author{
Jian-Bin Bao ${ }^{1,2 *}$ and Nicholas P. Bao ${ }^{3}$ \\ 1. Alumni Association, Zhejiang University, Hangzhou, Zhejiang 310027, P. R. China; \\ 2. Alumni Association, University of Alberta, Edmonton, Alberta T5J 4P6, Canada; \\ 3. Archbishop MacDonald Catholic High School, Edmonton, Alberta T5N 1H5, Canada \\ *E-mail: jbbao@ hotmail.com; jbao@ualberta.ca
}

\begin{abstract}
There are unsolved problems related to inflation, gravity, dark matter, dark energy, and the fate of the universe. Some of them can be better answered by assuming the existence of aether and hypoatoms. Both were created during the inflation in the very early universe. While aether forms vacuum, hypoatoms form all observable matter. In vacuum, aether exists between the particle-antiparticle form and the energy form in a dynamic equilibrium: $\mathrm{A}+\overline{\mathrm{A}} \rightleftharpoons \gamma+\gamma$, resulting in quantum phenomena and a character of negative pressure. The proposed hypoatom has an antimatter nucleus, with an equal mass of matter particles of aether in its perimeter, so the enigma of missing antimatter does not exist. At hypoatoms, the forward reaction of the aether annihilation dominates. With constantdensity dark energy, the annihilation constantly consumes the aether in vacuum, producing a sink flow of aether that warps spacetime, and thus generates gravity and a dark matter halo in the vicinity of massive objects. The hypoatom is believed to be a neutrino $v_{1}$, with a mass of $5 \mathrm{meV}$. Based on the hypoatom structure, singularities do not exist inside black holes; their cores are hypoatom stars or neutrino stars. By gaining enough mass, ca. $8 \times 10^{22} M_{\odot}$, to exceed neutrino degeneracy pressure, a black hole collapses or annihilates into the singularity, thus turning itself into a white hole or a new Big Bang.
\end{abstract}

Keywords: hypoatom, aether, inflation, gravity, negative pressure, dark energy, dark matter, neutrino oscillations, parity asymmetry, black holes, matter-antimatter asymmetry, the fabric of space, the fate of the universe

For the Schwarzschild metric:

$$
d s^{2}=-\left(1-\frac{2 G M}{r c^{2}}\right) c^{2} d t^{2}+\left(1-\frac{2 G M}{r c^{2}}\right)^{-1} d r^{2}+r^{2} d \Omega^{2}
$$

if we define speed

$$
v=\sqrt{2 G M / r}
$$

we have

$$
d s^{2}=-\left(1-\frac{v^{2}}{c^{2}}\right) c^{2} d t^{2}+\left(1-\frac{v^{2}}{c^{2}}\right)^{-1} d r^{2}+r^{2} d \Omega^{2}
$$

on the right of which, the first term shows the time dilation effect, and the second term shows the length contraction effect. This unequivocally indicates that the stationary objects in a gravitational field are in motion. The length contraction effect in equation (3) even 
says that the direction of motion is radial and the speed $v$ of motion is equal to the escape speed $v_{\text {esc }}=\sqrt{2 G M / r}$. Since motion is always relative to a frame of reference, we can make the stationary objects move by finding the reference frame that is moving in the gravitational field. We therefore believe that there exist fundamental particles. Einstein once stated": "according to the general theory of relativity, space without aether is unthinkable." We then use the term aether to name the fundamental particles.

Creation of fundamental particles. To know what aether could be, we start with the idea of the inflation that was proposed to resolve the horizon, flatness, and magnetic monopole problems ${ }^{2}$. Although the physical mechanism underlying inflation is still uncertain, it is often believed to be the result of a scalar field rolling down from the false vacuum to the true vacuum ${ }^{3,4}$. We study the inflation in a different way by proposing the following reactions in the very early universe:

$$
\begin{aligned}
& \mathrm{E} \rightarrow \mathrm{A}+\overline{\mathrm{A}} \\
& \mathrm{A}+\overline{\mathrm{A}} \rightarrow \mathrm{E} \\
& n \mathrm{~A}+n \overline{\mathrm{A}} \rightarrow \gamma+\gamma \\
& \gamma+\gamma \rightarrow \mathrm{B}+\overline{\mathrm{B}} \\
& \mathrm{B}+\overline{\mathrm{B}} \rightarrow \gamma+\gamma \\
& \mathrm{B}+n \overline{\mathrm{A}} \rightarrow \gamma+\gamma \\
& \overline{\mathrm{B}}+n \mathrm{~A} \rightarrow \gamma+\gamma
\end{aligned}
$$

Reaction (4) was the first phase transition in the universe, converting the pure energy (E) of the singularity universe into the particles $(\mathrm{A})$ and antiparticles $(\overline{\mathrm{A}})$ that are aether (Fig. 1). This reaction is based on Einstein's mass-energy equivalence; matter and antimatter pairs are the only possible products from energy. What the supercooled universe could first create could only be the most fundamental, even though it had sufficient energy to create any complicated particles. The fundamental aether particles $\mathrm{A}$ and $\overline{\mathrm{A}}$ settled at the lowest mass and in the smallest size with the highest stability possible for them to survive in the hot and dense primeval universe. As the reaction finished, the universe became a globe of aether particles $\mathrm{A}$ and $\overline{\mathrm{A}}$; the volume of the universe increased exponentially. This is the inflation. As the energy of the universe was used up, the temperature of the universe dropped significantly, and the inflation ended naturally.

Right after the creation, aether particles $A$ and $\bar{A}$ were annihilated, raising the temperature of the universe back to what the adiabatic standard cosmology describes (Fig. 1). This is known as the reheating process. In this work, we postulate: annihilation only happens if an equal mass of matter and antimatter particles are in contact with each other. Therefore, numerous annihilations initiated simultaneously across the globe of the universe, competing for the remaining aether particles $\mathrm{A}$ and $\overline{\mathrm{A}}$, and finished together when there were no more A and $\bar{A}$ left in contact. This process resulted in the maximum number of pairs ( $n$ pairs) of aether particles that were annihilated to produce photons (reaction 6 ), 
while a pair or a small number of pairs of $\mathrm{A}$ and $\overline{\mathrm{A}}$ particles were annihilated to energy (represented by reaction 5) because the wavelengths of products should not be larger than the size of the universe at that moment. It is reaction (6) that allowed the universe to deviate from energy and create more complicated particles (discussed below). Of course, the annihilations were impossible to be $100 \%$ complete. Some aether particles were left along and therefore survived.

The formed photons then collided to create particles B and antiparticles $\bar{B}$ (Fig.1) by the Breit-Wheeler pair production process (reaction 7). This phase transition has been regarded as the second episode of inflation ${ }^{5,6}$, though the universe would not experience a significant change of volume as it was already large. Immediately followed were three possible annihilations to reheat the universe: 1) $\mathrm{B}$ and $\overline{\mathrm{B}}$ (reaction 8 ); 2) $n \overline{\mathrm{A}}$ 's with a $\mathrm{B}$ (reaction 9, the total mass of matter must be equal to that of antimatter: $n m_{\overline{\mathrm{A}}}=m_{\mathrm{B}}$ ); and 3) $n$ A's with a $\overline{\mathrm{B}}$ (reaction $10, n m_{\mathrm{A}}=m_{\overline{\mathrm{B}}}$ ). Because the primeval universe was dense enough to allow multiple $\bar{A}$ 's to contact with a $\mathrm{B}$ simultaneously and multiple $\mathrm{A}$ 's to contact with a $\bar{B}$ simultaneously for annihilation, both reactions (9) and (10) were possible.

Although the reaction parameters are difficult to know, these proposed reactions provide a possible mechanism of the inflation and reheating. More importantly, it is the products of these reactions that formed the observable universe.

Hypoatom. Define the amount of reaction (9) as $N_{1}$, and the amount of reaction (10) as $N_{2}$. Once

$$
N_{1} \neq N_{2}
$$

an asymmetry appears. For simplicity of discussion in this work, let $N_{1}>N_{2}$ (in the other case $N_{1}<N_{2}$, the result is absolutely identical), then the leftovers were $\overline{\mathrm{B}}$ particles.

The survived $\overline{\mathrm{B}}$ particles recombined with aether particles A to form a species $\overline{\mathrm{B}} \mathrm{A}_{n}$. With the structure of an atom in mind, we believe that the $\overline{\mathrm{B}}$ is a nucleus and that $n \mathrm{~A}^{\prime} \mathrm{s}$ surround the $\overline{\mathrm{B}}$ (Fig. 2). We call $\overline{\mathrm{B}} \mathrm{A}_{n}$ a hypoatom.

Based on our postulate, the annihilation of a hypoatom only happens if all of the $n+1$ particles that form the hypoatom contact simultaneously (reaction 10). Because $n$ is large enough or $m_{\mathrm{A}}$ is small enough, hypoatoms $\overline{\mathrm{B}} \mathrm{A}_{n}$ are stable.

Fabric of space. Aether particles, as the fundamental units, formed vacuum ${ }^{7}$, or the fabric of space (Fig. 2). Since they are undetectable, whether A and $\bar{A}$ are bosons or fermions is unknown, so is the nature of interparticle interaction. However, it is safe to assume that their pairs are bosons and the interaction between $\mathrm{A}$ and $\overline{\mathrm{A}}$ is attractive. As it has expanded for a long time after the Big Bang, the universe cooled down to a temperature of $2.7 \mathrm{~K}$. Aether particles are much lighter than atoms, corresponding to a much higher transition temperature $^{8}$, so the fabric of space became a Bose-Einstein condensate (BEC) when it was hot. However, if the interparticle interaction is attractive, then an atomic BEC could become unstable and make particles clump together ${ }^{8}$. 
The fabric of space is different. Once a pair of $\mathrm{A}$ and $\overline{\mathrm{A}}$ attract to clump or contact, the following annihilation happens:

$$
\mathrm{A}+\overline{\mathrm{A}} \rightleftharpoons \gamma+\gamma
$$

The generated photons (the energy form of aether) have a superlong wavelength, because as the fundamental particles, $\mathrm{A}$ and $\overline{\mathrm{A}}$ have the smallest non-zero mass in nature. The superlong wavelength photons (SWP) can penetrate any matter in the universe, and eventually collide to create aether particles $\mathrm{A}$ and $\overline{\mathrm{A}}$ at an arbitrary distance away (the reverse of reaction 12). For reference, a reported mass ${ }^{9}, 8.1 \times 10^{-23} \mathrm{eV}$, could be a candidate of aether. For simplicity, similar processes such as the annihilation and creation between photons and particles will be omitted.

Due to reaction (12), as well as the induced collective oscillations of aether particles, the velocity of a specific aether particle in the fabric of space, as Dirac has foreseen, can no longer be well defined ${ }^{10}$. Absolute vacuum, or the pure fabric of space is a dynamic equilibrium system, with an equal rate of annihilation and creation of aether particles:

$$
R_{\mathrm{a}}=R_{\mathrm{c}}
$$

It is important to note that the rate $R_{\mathrm{a}}$ of the annihilation is controlled by the local concentration of aether particles, while the rate $R_{\mathrm{c}}$ of the creation is determined by the energy density of the whole fabric of space, i.e. the vacuum energy density.

Dark energy. Based on reaction (12), though the interaction between $\mathrm{A}$ and $\overline{\mathrm{A}}$ is attractive, the effective interaction ${ }^{8}$ is repulsive, and hence, the fabric of space is a stable system. Compared to the momenta of the oscillations, the mass of an A or $\overline{\mathrm{A}}$ is negligible: $m_{\mathrm{A}} \ll$ $q \hbar / c$, therefore the Bogoliubov dispersion relation is simplified ${ }^{11}$ :

$$
\omega=q c
$$

where $q, \hbar, c, \omega$ are wave vector, reduced Planck constant, speed of light, and angular frequency, respectively. Based on the Planck distribution, almost all of the oscillations occupy the ground state:

$$
\epsilon_{0}=\frac{1}{2} \hbar \omega
$$

Since the density of states is: $g(\omega)=V q^{2} / 2 \pi^{2} c$, we have the vacuum energy density:

$$
\varepsilon_{\mathrm{vac}}=\frac{1}{V} \int_{0}^{\omega_{\mathrm{A}, \mathrm{m}}} g(\omega) \epsilon_{0} d \omega=\frac{\hbar \omega_{A, \mathrm{~m}}^{4}}{16 \pi^{2} c^{3}}
$$

where $\omega_{\mathrm{A}, \mathrm{m}}$ is the maximum angular frequency. As aether particles are oscillating in equilibrium with hypoatoms:

$$
\hbar^{2} \omega_{\mathrm{A}, \mathrm{m}}^{2}=m_{\overline{\mathrm{B}} \mathrm{A}_{\mathrm{n}}}^{2} c^{4}+q_{\overline{\mathrm{B}} \mathrm{A}_{\mathrm{n}}}^{2} c^{2} \hbar^{2} \approx m_{\overline{\mathrm{B}} \mathrm{A}_{\mathrm{n}}}^{2} c^{4}
$$

the vacuum energy density is

$$
\varepsilon_{\mathrm{vac}} \approx \frac{m_{\mathrm{BA}}^{4} c^{5}}{16 \pi^{2} \hbar^{3}}
$$

According to the Planck 2018 results $^{12}$, Hubble constant $H_{0}=67.66 \pm$ $0.42 \mathrm{~km} \mathrm{~s}^{-1} \mathrm{Mpc}^{-1}$, the density parameter for dark energy $\Omega_{\Lambda}=0.6889 \pm 0.0056$, so the 
vacuum energy density is $5.92 \times 10^{-27} \mathrm{~kg} / \mathrm{m}^{3}$. Therefore, the upper limit of the mass of the hypoatom is ca. $8 \mathrm{meV}$.

Neutrino. This mass is close to that of neutrinos. With the concept of the hypoatom in mind, we assume that neutrinos are composed of hypoatoms: $v_{1}=\left(\overline{\mathrm{B}} \mathrm{A}_{n}\right)_{a}, v_{2}=$ $\left(\overline{\mathrm{B}} \mathrm{A}_{n}\right)_{b}$, and $v_{3}=\left(\overline{\mathrm{B}} \mathrm{A}_{n}\right)_{c}, n \gg 1 ; n, a, b, c \in N$. From the neutrino oscillation, obtained are the mass squared differences of the three mass-eigenvalue neutrinos: $\Delta m_{21}^{2} \equiv m_{2}^{2}-$ $m_{1}^{2}=7.50_{-0.17}^{+0.19} \times 10^{-5} \mathrm{eV}^{2},\left|\Delta m_{31}^{2}\right| \equiv\left|m_{3}^{2}-m_{1}^{2}\right|=2.457_{-0.047}^{+0.047} \times 10^{-3} \mathrm{eV}^{2}$, where $\Delta m_{31}^{2} \equiv \Delta m_{31}^{2}>0$ for the normal ordering (NO) and $\Delta m_{31}^{2} \equiv \Delta m_{32}^{2}<0$ for the inverted ordering $(\mathrm{IO})^{13}$; and $\Delta m_{21}^{2}=(7.53 \pm 0.18) \times 10^{-5} \mathrm{eV}^{2}, \Delta m_{32}^{2}=(2.453 \pm 0.034) \times$ $10^{-3} \mathrm{eV}^{2}$ for the $\mathrm{NO}$, and $\Delta m_{32}^{2}=\left(-2.546_{-0.040}^{+0.034} \pm 0.07\right) \times 10^{-3} \mathrm{eV}^{2}$ for the $\mathrm{IO}^{14}$. If we assume that the lightest neutrino is a hypoatom and the interaction energy between hypoatoms is negligible, then for the NO,

$$
\begin{aligned}
& m_{1} \approx 5 \mathrm{meV}, a=1 \\
& m_{2} \approx 10 \mathrm{meV}, b=2 \\
& m_{3} \approx 50 \mathrm{meV}, c=10
\end{aligned}
$$

and for the IO,

$$
\begin{aligned}
& m_{1} \approx 49.4 \mathrm{meV}, a=65 \\
& m_{2} \approx 50.2 \mathrm{meV}, b=66 \\
& m_{3} \approx 0.76 \mathrm{meV}, c=1
\end{aligned}
$$

While the existence of the oscillation between 65 and 66 hypoatoms is hard to imagine, the NO is much more reasonable as the ratios of $b / a, c / a$, and $c / b$ for the NO are all integers. In addition, the amplitude of neutrino oscillation can be characterized by $\sin ^{2}(2 \theta)$, where $\theta$ is the mixing angle. Since $\sin ^{2}\left(2 \theta_{13}\right)=0.0853$ is smaller than $\sin ^{2}\left(2 \theta_{12}\right)=0.851$ and $\sin ^{2}\left(2 \theta_{23}\right)=0.991$ (calculated from ref. 14), the probability of the oscillation between $v_{1}$ and $v_{3}$ is the smallest, which also favors the NO. The newest fitting on experimental data also indicates that the possibility of the NO is larger than that of the $\mathrm{IO}^{15}$. We thus conclude: a) neutrinos are composed of hypoatoms; b) neutrino oscillation is the quantum interference of the rearrangement of hypoatoms; c) the mass of a hypoatom is ca. $5 \mathrm{meV}$.

The experimental data in the literature support our conclusions. For example, if $\overline{\mathrm{B}} \mathrm{A}_{n}$ is a neutrino, then $\mathrm{B}_{\overline{\mathrm{A}}}$ is an antineutrino. Now if we measure the cross section of antineutrinos, using a matter detector of course, aether antiparticles $\bar{A}$ in the perimeter of the antineutrinos annihilate with aether particles $\mathrm{A}$ in the perimeter of the matter detector, thus the obtained cross section is reduced. On the contrary, when we measure the cross section of neutrinos, both neutrinos and detectors have the same perimeter particles $\mathrm{A}$. Therefore, the cross section of antineutrino $\mathrm{B} \overline{\mathrm{A}}_{n}$ is always smaller than that of neutrino $\overline{\mathrm{B}} \mathrm{A}_{n}$. For comparison, numerous experimental data have shown that the cross sections of neutrinos are twice as large as those of corresponding antineutrinos ${ }^{14}$. A recent effort has 
directly observed matter-antimatter asymmetry in neutrino oscillation ${ }^{16}$. Based on the proposed structure of hypoatoms, such asymmetry is natural.

Obviously, if hypoatoms $v_{1}$ can form $v_{2}$ and $v_{3}$, there is no reason that hypoatoms cannot form other larger particles in the Standard Model of particle physics, or strings, as atoms form polymer.

Now we have a sketch of the universe: a) the fabric of space, or vacuum, is formed by aether particles $A$ and $\bar{A}$, and SWP, the energy form of aether; b) all observable particles or matter is composed of hypoatoms $\overline{\mathrm{B}} \mathrm{A}_{n}$. Because antiparticles $\overline{\mathrm{B}}$ are hidden by aether particles $\mathrm{A}$ in hypoatoms, the enigma of missing antimatter does not exist! It is of interest to note from its composition that a hypoatom $\overline{\mathrm{B}} \mathrm{A}_{n}$ does own the energy that Einstein taught us: $E=m_{\overline{\mathrm{B}} A_{n}} c^{2}$, and it can be released once annihilated. A typical example is the nuclear energy that is released when some hypoatoms or neutrinos are annihilated in nuclear reactions.

General theory of relativity. In the general theory of relativity, the pure fabric of space is regarded as flat spacetime:

$$
d s^{2}=-c^{2} d t^{2}+d r^{2}+r^{2} d \Omega^{2}
$$

Based on our proposed model, the annihilation and creation of aether are in equilibrium (reaction 12). Therefore, there is no net flow of aether particles (equation 13), and hence, aether has no observable mass in the pure fabric of space.

Now if a massive object is introduced, the rate $R_{\mathrm{a}}$ of annihilation rises dramatically. An oscillating aether antiparticle $\bar{A}$ from the fabric of space annihilates easily with one of the $n$ aether particles $\mathrm{A}$ at the perimeter of a hypoatom $\overline{\mathrm{B}} \mathrm{A}_{n}$ (step 1, Fig. 2):

$$
\overline{\mathrm{B}} \mathrm{A}_{n}+\overline{\mathrm{A}} \rightarrow\left[\overline{\mathrm{B}} \mathrm{A}_{n-1}\right]+\gamma+\gamma
$$

The vacancy left in the hypoatom will then be filled by a particle $A$ from the fabric of space (step 2, Fig. 2):

$$
\left[\overline{\mathrm{B}} \mathrm{A}_{n-1}\right]+\mathrm{A} \rightarrow \overline{\mathrm{B}} \mathrm{A}_{n}
$$

As the fabric of space is oscillating constantly, hypoatoms $\overline{\mathrm{B}} \mathrm{A}_{n}$ act like catalysts in chemical reactions, consuming the aether particles in the fabric of space and converting them into SWP:

$$
\mathrm{A}+\overline{\mathrm{A}} \rightarrow \gamma+\gamma
$$

This annihilation generates a 3-dimensional sink flow of aether particles towards the center of the massive object. It is this sink flow that warps the spacetime in the vicinity of the massive object! Because of this flow, the time term of equation (21) needs to be rewritten with the time-dilation expression: $\sqrt{1-v^{2} / c^{2}} d t$ and its radial term with the lengthcontraction expression: $\frac{d r}{\sqrt{1-v^{2} / c^{2}}}$, while the last term on the right stays $\operatorname{correct}^{17}$. Considering equation (2), we have the Schwarzschild metric (equation 1). To the best of our knowledge, this is the first derivation of the general theory of relativity, the greatest law of nature. If the speed of the sink flow is small, the relativistic effect is negligible; we 
then have Gauss's law or Poisson's equation for gravity: $\nabla^{2} \phi=4 \pi G \rho$, equivalent to Newton's law of universal gravitation.

In the above analysis, the creation of aether particles (the reverse of reaction 12), which reduces the sink flow a bit, has not been considered, because compared to that of the annihilation, the rate of the creation is negligible:

$$
R_{\mathrm{a}}-R_{\mathrm{c}} \approx R_{\mathrm{a}}
$$

If it is considered, then Einstein's field equation should be written with the addition of the $\Lambda$ term, and Newton's law is: $\nabla^{2} \phi=4 \pi G \rho-\Lambda c^{2}$.

With the existence of the sink flow, aether particles in the fabric of space are in fact oscillating in quasi-equilibrium with hypoatoms (equation 17). It is time to note that this quasi-equilibrium is natural: if the vacuum energy density were higher, then more aether particles would be annihilated at hypoatoms, equivalent to a higher flowrate of the sink flow, thus resulting in a larger hypoatom mass and vice versa.

Based on the Landau criterion, within the limit of the speed of light (equation 14), the fabric of space is a superfluid ${ }^{7}$. Therefore, the sink flow only generates the curvature of spacetime, but does not pull or push objects directly.

Interestingly, our model does show that the fabric of space has the character of negative pressure that is vital to Einstein's field equation. Think about a cylinder-piston system. When $\mathrm{A}$ and $\overline{\mathrm{A}}$ are created (the reverse of reaction 12), the change of internal energy is: $\Delta U=m c^{2}$, where $m$ is the mass of aether particles $\mathrm{A}$ and $\overline{\mathrm{A}}$ created. The creation of aether particles increases the volume of the system, so the system does work: $W=p \Delta V$. According to the first law of thermodynamics, we have $m c^{2}=-p \Delta V$ or $p=$ $-\rho c^{2}$. When $\mathrm{A}$ and $\overline{\mathrm{A}}$ are annihilated (the forward of reaction 12), the character is identical. The negative pressure can also be understood in a different way. Each time when an $\bar{A}$ from the fabric of space annihilates with an aether particle A of a hypoatom (reaction 22), the hypoatom temporarily loses, not gains, the momentum of the annihilated aether particle A. Therefore, the hypoatom experiences negative pressure.

Einstein taught us that the curvature of spacetime causes gravity, but did not mention why. Using the character of negative pressure, it is not difficult to understand gravity. For an object $m$ under the curvature of spacetime generated by an object $M$, the annihilation rate on the side near $M$ is higher than that on the far side. In other words, the force on the near side (pointing towards $M$ ) is larger than that on the far side (pointing away from $M$ ). Therefore, $m$ experiences an attractive force, which is gravity.

Newton taught us that gravity is universal. Indeed it is, because all matter is made of hypoatoms. For the same reason, gravity cannot be shielded by any objects. The objects in the gravitational field do nothing but result in reaction (24), further warping spacetime. This is also true for energy, which enhances the annihilation of aether particles and generates gravity, in agreement with the general theory of relativity. 
The difficulty of unifying four fundamental forces is well known. Based on our model, gravity, in fact, is a collective property of aether particles in the fabric of space, while the other three forces are just interactions of the interested particles or objects.

Dark matter. For a uniform sphere $M$ of radius $r_{M}$ and density $\rho_{\mathrm{M}}$, the rate $R_{a}$ of annihilation can be calculated by the flowrate of the sink flow:

$$
R_{a}=4 \pi r^{2} v_{\mathrm{esc}} \rho_{\mathrm{A}}
$$

where $A$ is the mass of the aether particles within the sphere of radius $r$, and $\rho_{\mathrm{A}}$ the density of the aether particles. The rate of annihilation is also proportional to mass:

$$
R_{a}=k(M+A)
$$

where $k$ is the proportional constant, then we have

$$
d A=\rho_{\mathrm{A}} \cdot 4 \pi r^{2} d r=\left(\frac{k^{2}}{2 G}\right)^{\frac{1}{2}}(M+A)^{\frac{1}{2}} r^{\frac{1}{2}} d r
$$

By integration, the mass of aether is

$$
A=\left(\frac{2 k^{2} M}{9 G}\right)^{\frac{1}{2}} r^{\frac{3}{2}}+\frac{k^{2}}{18 G} r^{3}
$$

and the density of aether is

$$
\rho_{\mathrm{A}}=\left(\frac{k^{2} M}{32 \pi^{2} G}\right)^{\frac{1}{2}} r^{-\frac{3}{2}}+\frac{k^{2}}{24 \pi G}
$$

The last term on the right is the density at infinite distance $(r \rightarrow \infty): \rho_{\mathrm{A}, \infty} \equiv \frac{k^{2}}{24 \pi G}$, which is also the density in the pure fabric of space. By subtracting it, the density $\rho_{\mathrm{D}}$ of dark matter is

$$
\rho_{\mathrm{D}}=\rho_{\mathrm{A}}-\rho_{\mathrm{A}, \infty}=\left(\rho_{\mathrm{M}} \rho_{\mathrm{A}, \infty}\right)^{\frac{1}{2}}\left(\frac{r_{\mathrm{M}}}{r}\right)^{\frac{3}{2}}
$$

The mass $D$ of dark matter in the vicinity of $M$ is

$$
D=2\left(\frac{4}{3} \pi r^{3} \rho_{\mathrm{A}, \infty} M\right)^{\frac{1}{2}}
$$

which is divergent. This indicates that all the halos in the universe are overlapping, which is correct as gravity is infinite. To estimate the major part of dark matter, we need to choose a cutoff radius. From $-\frac{G M}{r}+\frac{1}{6} \Lambda c^{2} r^{2}=0$, where $\Lambda=8 \pi G \rho_{\mathrm{vac}} / c^{2}$, and $\rho_{\mathrm{vac}}$ is the density of dark energy, we have

$$
r_{\text {cut-off }}^{3}=\frac{6 G M}{\Lambda c^{2}}=\frac{M}{\frac{4}{3} \pi \rho_{\mathrm{vac}}}
$$

The mass of dark matter in the vicinity of $M$, at least, is

$$
D_{\text {min }}=2\left(\frac{\rho_{\mathrm{A}, \infty}}{\rho_{\mathrm{vac}}}\right)^{\frac{1}{2}} M
$$

The most important piece of information here is that every massive object in the universe has a dark matter halo whose mass is proportional to its own mass. Because the ratio $\rho_{\mathrm{vac}} / \rho_{\mathrm{A}, \infty}$ or the equilibrium constant of reaction (12) is unknown, we cannot compare $D_{\min } / M$ with the ratio of dark matter to ordinary matter from cosmological 
observation. Given the fact that most popular stellar systems are galaxies composed of stars, this proportional relation allows us to estimate the mass of the dark matter halo for a galaxy by simply summing up all the masses in the galaxy. Because stars are distributed in the galaxy, the density will reduce along the radial direction a little slower than that of an isolated object ( $r^{-1.5}$, equation 31$)$. For comparison, a profile ${ }^{18}$ of dark matter obtained from cosmological $N$-body simulations has an asymptotic slope of $r^{-1.4}$. Therefore, aether is a major contributor to dark matter.

It is of interest to note that as the universe expands, the mass ratio of dark matter to ordinary matter almost stays the same (equations 32 and 34), while the mass ratio of dark energy to matter increases with the expansion because the density of dark energy is a constant (equation 18). This is in agreement with the results of cosmological observation.

Quantum mechanics. Based on our model, quantum mechanics can be understood relatively easily. Here are a couple of examples. Because all particles are in the fabric of space and the fabric of space is a constantly oscillating dynamic system, the wave-particle duality is natural. The zero-point vibration of aether particles (equation 15) is equivalent to Heisenberg's uncertainty principle. In theory, particles heavier than aether could be created by multiple SWP. Of course, the heavier the particles, the lower the chance of creation, corresponding to a shorter lifetime in the quantum field theory. The wave function of a particle from the Schrodinger equation is the superposition of wave vectors from the local interactions and the creation and annihilation of aether particles in the fabric of space, not a property of only the interested particle itself. This is why Lorentz invariance is violated for Schrodinger's wave functions. For the double-slit interference experiment, the interference pattern of electrons (or photons) is obtained because the oscillation of the local fabric of space is interfered by the slits. When they are observed, the light of observation overwrites the interfered fabric of space, or the so-called wave function collapse occurs, hence the interference pattern no longer exists.

Another unsolved problem is the conflict between the general theory of relativity and quantum mechanics. For a massive object, as the size goes down, for example, to the Schwarzschild radius, quantum effects from the dynamic fabric of space become negligible. Therefore, the general theory of relativity and quantum mechanics are compatible.

An earlier inflation. The inflation is generally assumed in the context of the Grand Unified Theory (GUT) $)^{2,3,4,19}$, though a wider range was proposed ${ }^{20}$. While compatible with a start at $10^{-34} \mathrm{sec}$ after the Big Bang or $10^{16} \mathrm{GeV}$ of GUT energy scale, our model suggests that the inflation started as soon as the Planck time. This is because: a) reaction (4) converted the pure energy of the singularity into aether particles $\mathrm{A}$ and $\overline{\mathrm{A}}$; b) $\mathrm{A}$ and $\overline{\mathrm{A}}$ are not included in the GUT; c) it is widely accepted that gravity begins to differentiate from the other three forces at the Planck time. 
At the Planck time, $5.4 \times 10^{-44} \mathrm{sec}$, the energy density of the false vacuum was $1.2 \times 10^{19} \mathrm{GeV}$, equivalent to the mass density of $5.2 \times 10^{96} \mathrm{~kg} / \mathrm{cm}^{3}$. The inflation started at a patch size of the Planck length, $l_{\mathrm{Pl}}=1.6 \times 10^{-35} \mathrm{~m}$. From the first-order Friedman equation: $\left(\frac{\dot{a}}{a}\right)^{2}=\frac{8 \pi G \rho_{f}}{3}$, we have the size of the universe exponentially increase in the function of $a=$ Constant $\cdot \exp (\chi \mathrm{t})$, where $\chi=\sqrt{\frac{8 \pi G \rho_{f}}{3}}=\sqrt{\frac{8 \pi c^{5}}{3 \hbar G}}=5.4 \times 10^{43} \mathrm{sec}^{-1}$. The universe after the first episode of the inflation and reheating (reactions $4 \sim 6$ ) would at least be as large as one half the wavelength of the B particle:

$$
a \approx \lambda_{\mathrm{B}} / 2 \approx \lambda_{v_{1}}=\frac{h c}{E_{v_{1}}}=0.25 \mathrm{~mm}
$$

Thus the size of the present universe:

$$
a_{0} \approx \frac{1.2 \times 10^{18}}{k_{\mathrm{B}} \times 2.7} \times 0.25 \mathrm{~mm}=1.4 \times 10^{11} \text { light years }
$$

which is in agreement with the inflationary theory ${ }^{19}$.

Interestingly, the first episode of the inflation (reaction 4) was a process to create, not consume, aether particles $\mathrm{A}$ and $\overline{\mathrm{A}}$. Therefore, the gravity during the inflation appeared as a repulsive force! Although producing an attractive gravity later on, $\mathrm{A}$ and $\overline{\mathrm{A}}$ in the inflation were a repulsive-gravity material. Based on earlier discussion, this process did produce negative pressure. This mechanism is in agreement with the inflationary theory ${ }^{19}$. By assuming an earlier inflation, we believe that at ca. $10^{-34} \sim 10^{-32} \mathrm{sec}$ there was another phase transition for the formation of particles $\overline{\mathrm{B}}$ or hypoatoms $\overline{\mathrm{B}} \mathrm{A}_{n}$, as neutrinos are the GUT particles.

Fate or birth of the universe. The most condensed star observed is a neutron star. If its mass further increases, it will collapse into a black hole. Within a black hole, it is believed to form a singularity. However, based on the proposed hypoatom structure, hypoatom degeneracy pressure can provide new support to prevent the collapse. In other words, the core of a black hole is indeed a hypoatom star or a neutrino star.

As the fabric of space is getting closer to a massive object, the density of aether increases (equation 31), so does the possibility of the annihilation of aether particles. For a black hole, as aether particles pass through the event horizon, the photons from the annihilation can no longer escape, so the temperature within the event horizon must be high. Fig. 3 illustrates the structure of a black hole. There naturally exists a fireball surrounding its hot core. This annihilation process could easily become a nonlinear system, leading to pulsations or the so-called quasi-periodic oscillation.

Analogous to the Tolman-Oppenheimer-Volkoff limit for neutron stars, we can estimate a mass limit for a neutrino star or a black hole:

$$
M \approx\left(\frac{m_{\mathrm{n}}}{m_{v_{1}}}\right)^{2} M_{\mathrm{n}} \approx\left(\frac{939.6 \mathrm{MeV}}{5 \mathrm{meV}}\right)^{2} \times 2.2 M_{\odot}=8 \times 10^{22} M_{\odot}
$$


where $m_{\mathrm{n}}$ is the mass of a neutron, $m_{v_{1}}$ the mass of $v_{1}, M_{\mathrm{n}}$ the mass limit of a neutron star, and $M_{\odot}$ the mass of the sun. Without considering a potential addition due to the rotation of the black hole, this mass limit is very close to the accepted mass of the observable universe, ca. $10^{23} M_{\odot}$. If the mass of a black hole exceeds this limit, the structure of the hypoatoms cannot withhold and thus the black hole or neutrino star collapses. In result, all aether particles $\mathrm{A}$ and antiparticles $\overline{\mathrm{B}}$ annihilate to become the pure energy (no space for photons) that is named as a singularity. Thus a black hole turns into a white hole; a new Big Bang begins (Fig. 4).

The lowest cross section of neutrinos experimentally measured ${ }^{21}$ is about $10^{-56} \mathrm{~cm}^{2}$. If we use it to estimate the density of the hypoatoms: $\rho_{v}=$ $5 \mathrm{meV} /\left(10^{-30} \mathrm{~m}\right)^{3} \sim 10^{52} \mathrm{~kg} / \mathrm{m}^{3}$, then the size of the black holes before the collapse can be estimated: $a \sim \sqrt[3]{m / \rho_{v}}=3 \mathrm{~m}$.

This collapse before the Big Bang can almost be regarded as the reverse process of the inflation after the Big Bang (Fig. 4). It is not difficult to believe that equation (11) is valid, due to the heritage of the previous generation, or a mechanism similar to the orbitspin interactions, as black holes usually have angular momenta or charges (the no-hair theorem). During this process, the particles to potentially form right-handed antineutrinos were annihilated (reaction 9), which would be the origin of the parity asymmetry that Lee and Yang discovered ${ }^{22}$. We further assume that the interactions of $\mathrm{A}$ and B particles would be related to weak force.

Based on our model, the universe is an open system. Given the fact that galaxies usually have supermassive black holes at their centers, the fate of the universe will end up with many new Big Bangs. This naturally explains the accelerating expansion of the current universe. Black holes, or their mergers, are the seeds of the universes of the next generation, and hence require space or energy to grow, though not all growth is guaranteed. Our universe is still at her young age!

\section{Acknowledgements}

J.B.B. would like to thank Profs. Z. Shen, K. Yao, X. Jiang, F. Bo, Y. Cheng, S. Han, G. Chen, and Q. Yu at Zhejiang University for their inspiration. N.P.B. would like to dedicate this work to his grandparents in China.

\section{References}

1. Einstein, A. Ether and the theory of relativity (Address Univ. Leiden, 5 May 1920).

2. Guth, A. H. Inflationary universe: a possible solution to the horizon and flatness problems. Phys. Rev. D 23, 347-356 (1981).

3. Linde, A. D. A new inflationary universe scenario: a possible solution of the horizon, flatness, homogeneity, isotropy and primordial monopole problems. Phys. Lett. 108B, 389-393 (1982).

4. Kolb, E. W. \& Turner, M. S. The early universe (Addison-Wesley, Redwood, 1990). 
5. Starobinsky, A. A. Multicomponent de Sitter (inflationary) stages and the generation of perturbations. JETP Lett. 42, 152-155 (1985).

6. Silk, J. \& Turner, M. S. Double inflation. Phys. Rev. D 35, 419-428 (1987).

7. Sinha, K. P., Sivaram, C. \& Sudarshan, E. C. G. Aether as a superfluid state of particle-antiparticle pairs. Found. Phys. 6, 65-70 (1976).

8. Pethick, C. J. \& Smith, H. Bose-Einstein condensation in dilute gases (Cambridge Univ. Press, Cambridge, 2002).

9. Schive, H.-Y., Chiueh, T. \& Broadhurst, T. Cosmic structure as the quantum interference of a coherent dark wave. Nat. Phys. 10, 496-499 (2014).

10. Dirac, P. A. M. Is there an aether? Nature 168, 906-907 (1951).

11. Fagnocchi S. et al. Relativistic Bose-Einstein condensates: a new system for analogue models of gravity. New J. Phys. 12, 095012 (2010).

12. Planck Collaboration: Aghanim N. et al. Planck 2018 results. VI. cosmological parameters. Astron. Astrophys. 641, A6 (2020).

13. Gonzalez-Garcia, M. C., Maltoni, M. \& Schwetz, T. Updated fit to three neutrino mixing: status of leptonic CP violation. J. High Energy Phys. 2014, 052 (2014).

14. Zyla P. A. et al. (Particle Data Group). Prog. Theor. Exp. Phys. 2020, 083C01 (2020).

15. Xing, Z. Z. Neutrino Oscillation (Shanghai Sci. Tech. Edu. Press, Shanghai, 2019).

16. The T2K Collaboration. Constraint on the matter-antimatter symmetry-violating phase in neutrino oscillations. Nature 580, 339-344 (2020).

17. Taylor, E. F., Wheeler, J. A. \& Bertschinger, E. Exploring black holes: introduction to general relativity. 2nd ed. (Addison-Wesley, Redwood, 2010).

18. Moore, B. et al. Resolving the structure of cold dark matter halos. Astrophys. J. 499, L5-L8 (1998).

19. Guth, A. H. Lecture 23: inflation (MIT Open Course Ware, 2013).

20. Linde, A. D. The inflationary universe. Rep. Prog. Phys. 47, 925-986 (1984).

21. Perkins, D. Particle Astrophysics. 2nd ed. (Oxford Univ. Press, New York, 2003).

22. Lee, T. D. \& Yang, C. N. Question of Parity Conservation in Weak Interactions. Phys. Rev. 104, 254-258 (1956). 


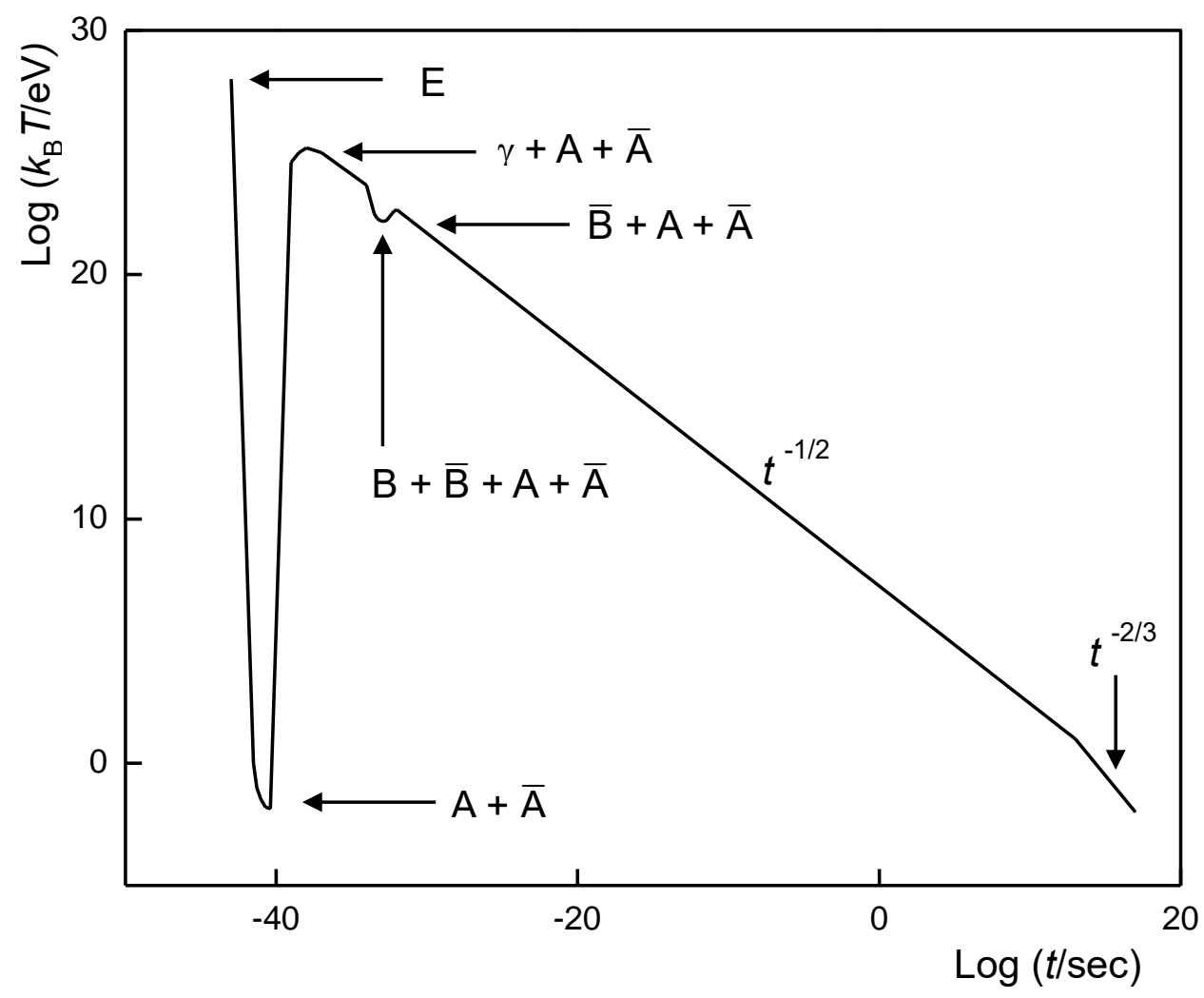

Fig. 1. Creation of the fundamental particles of nature. a) First episode of inflation: the pure energy (E) of the singularity turned into a globe of $\mathrm{A}$ and $\overline{\mathrm{A}}$ (reaction 4). The lowest temperature is taken from ref. 4. b) First reheating: numerous annihilations of $\mathrm{A}$ and $\overline{\mathrm{A}}$ raised the temperature back to what the adiabatic standard cosmology describes. While one or a small number of aether pairs annihilated into energy (represented by reaction 5), $n$ pairs of aether, by competition, produced photons (reaction 6). Some aether particles were left along and therefore survived. c) Second episode of inflation: the photons collided to create particles $\mathrm{B}$ and antiparticles $\overline{\mathrm{B}}$ (reaction 7). The temperature drop in this episode is much smaller than that in the first episode. Second reheating: the annihilations of particles $\mathrm{B}$ and $\overline{\mathrm{B}}$, without and with aether particles, raised the temperature again. Among them, the annihilated number of B with $\bar{A}$ 's (reaction 9 ) is assumed to be more than that of $\bar{B}$ with A's (reaction 10), thus particles $\overline{\mathrm{B}}$ were leftover to form hypoatoms $\overline{\mathrm{B}} \mathrm{A}_{n}$. 

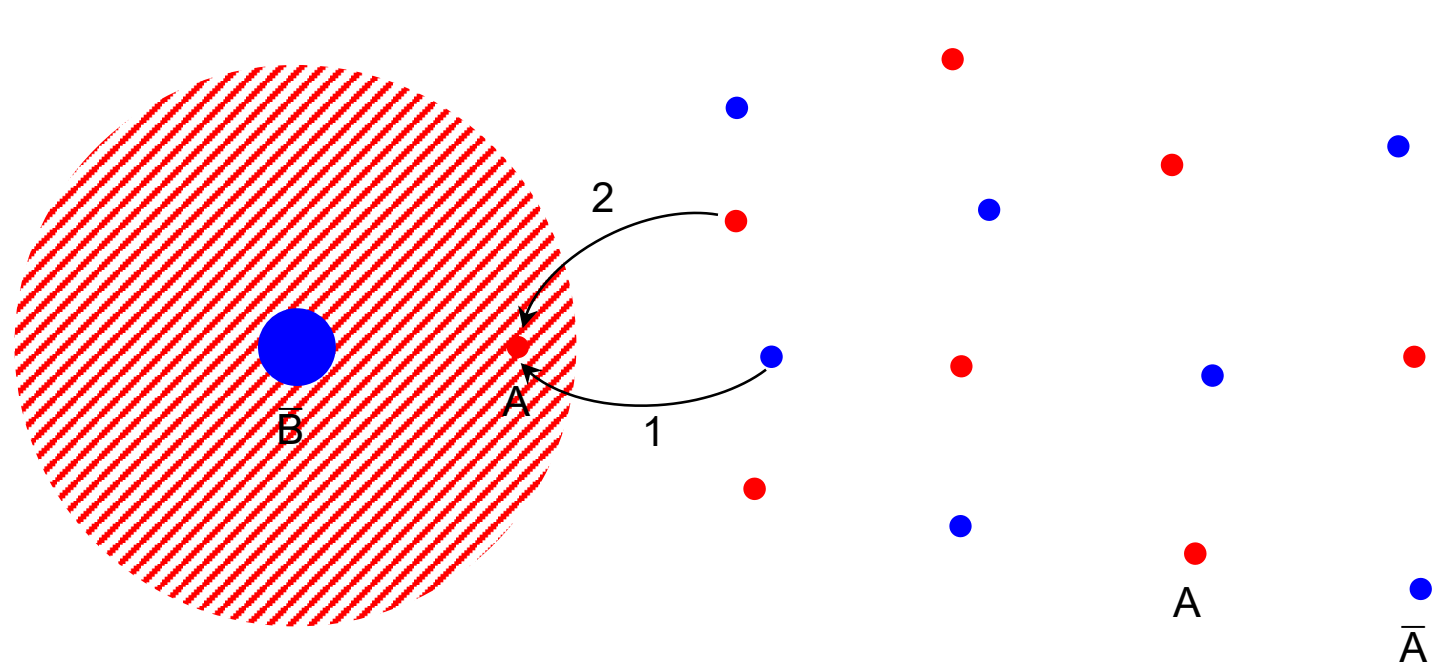

Fig. 2. A schematic diagram of a hypoatom, the fabric of space, and the generation of the sink flow of aether. Hypoatom $\overline{\mathrm{B}} \mathrm{A}_{n}$ : a particle $\overline{\mathrm{B}}$ (blue) is the nucleus; $n$ particles $\mathrm{A}$ (slashed red area) surround the particle $\overline{\mathrm{B}}$. Fabric of space: composed of aether particles $\mathrm{A}$ (red) and $\overline{\mathrm{A}}$ (blue). Sink flow: an aether particle $\overline{\mathrm{A}}$ (blue) in the fabric of space oscillates and contacts with an aether particle A (red) at the perimeter of the hypoatom and gets annihilated (step 1). The vacancy is then filled by another aether particle A (red) in the fabric of space (step 2). The hypoatom $\overline{\mathrm{B}} \mathrm{A}_{n}$ constantly annihilates the aether particles, converting them into SWP, and thus produces a 3-dimensional sink flow of aether, warping spacetime and generating gravity. 
Fig. 3. A schematic diagram of a black hole. The core (red) is a hot hypoatom star or neutrino star. The dash line (orange) is the event horizon. A fire (pink) is lit because of the annihilation of aether particles $\mathrm{A}$ and $\overline{\mathrm{A}}$. The intensity of the fire inside the event horizon is much higher than that outside the event horizon. If its mass exceeds the limit that the hypoatom degeneracy pressure can support, then the core collapses into a singularity. 


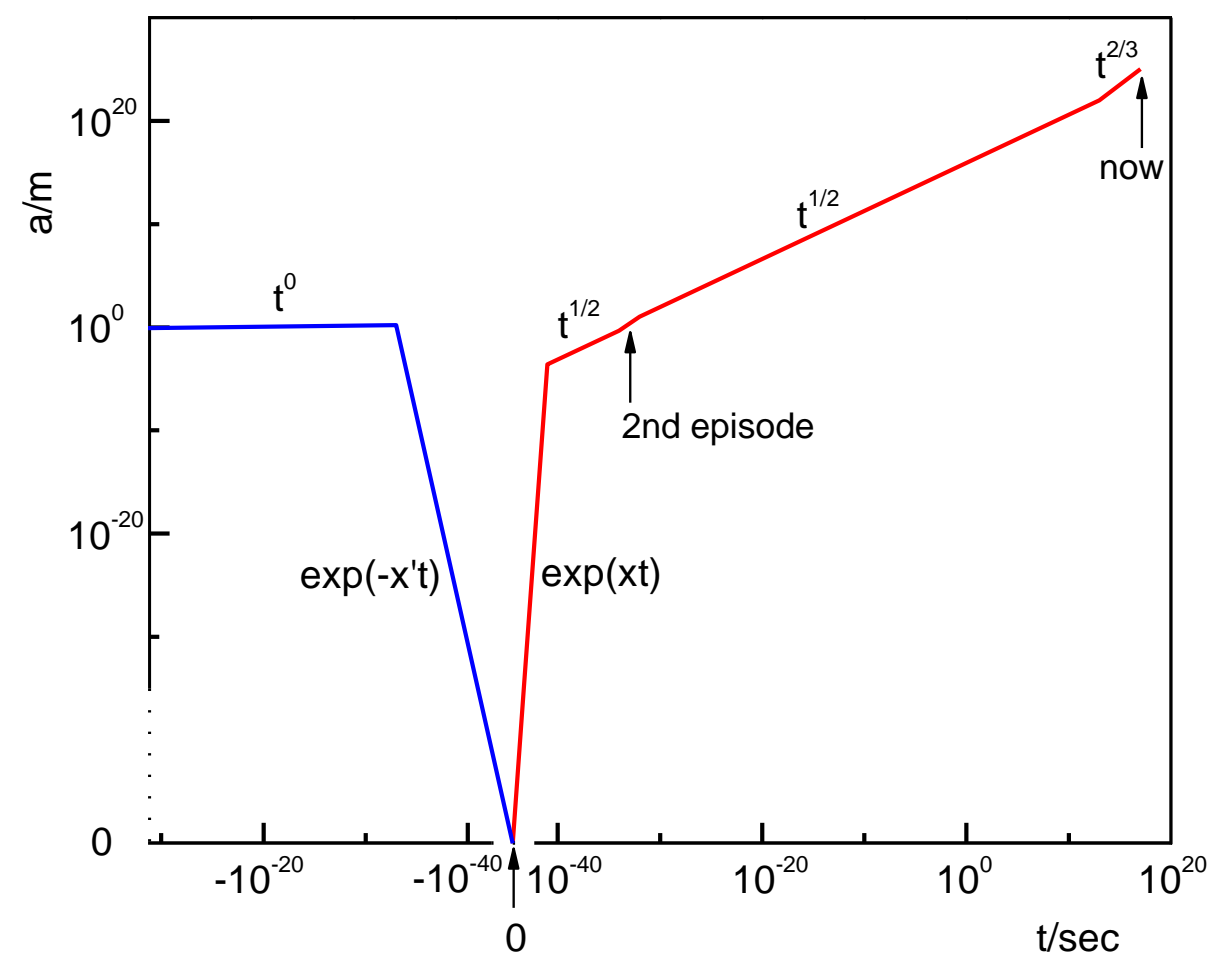

Fig. 4. The size of a universe before and after a Big Bang. Before the Big Bang (blue): it is a growing black hole. At ca. $10^{-34} \sim 10^{-32} \mathrm{sec}$ before the Big Bang when its mass exceeds ca. $8 \times 10^{22} M_{\odot}$, the core of the black hole collapses from ca. $3 \mathrm{~m}$ into a singularity. After the Big Bang (red): the universe expands with inflation and reheating processes, creating the fundamental particles of nature. After the first episode of inflation and reheating at ca. $10^{-41} \mathrm{sec}$, the size of the universe is ca. $0.25 \mathrm{~mm}$. After the second episode of inflation and reheating at ca. $10^{-34} \sim 10^{-32} \mathrm{sec}$, the universe returns to its size before the collapse. 